\title{
Quantification, improvement, and harmonization of small lesion detection with state-of-the-art PET
}

\author{
Charlotte S. van der Vos ${ }^{1,2}$ • Daniëlle Koopman ${ }^{2,3}$ • Sjoerd Rijnsdorp ${ }^{4}$. \\ Albert J. Arends ${ }^{4}$ - Ronald Boellaard ${ }^{5,6}$ • Jorn A. van Dalen ${ }^{3,7}$ • Mark Lubberink ${ }^{8,9}$ • \\ Antoon T. M. Willemsen ${ }^{5}$ Eric P. Visser ${ }^{1}$
}

Received: 4 May 2017 / Accepted: 9 May 2017 / Published online: 8 July 2017

(C) The Author(s) 2017. This article is an open access publication

\begin{abstract}
In recent years, there have been multiple advances in positron emission tomography/computed tomography (PET/CT) that improve cancer imaging. The present generation of PET/CT scanners introduces new hardware, software, and acquisition methods. This review describes these new developments, which include time-of-flight (TOF), pointspread-function (PSF), maximum-a-posteriori (MAP) based reconstruction, smaller voxels, respiratory gating, metal artefact reduction, and administration of quadratic weightdependent ${ }^{18} \mathrm{~F}$-fluorodeoxyglucose (FDG) activity. Also, hardware developments such as continuous bed motion (CBM), (digital) solid-state photodetectors and combined PET and magnetic resonance (MR) systems are explained. These novel techniques have a significant impact on cancer imaging, as they result in better image quality, improved small lesion detectability, and more accurate quantification of radiopharmaceutical uptake. This influences cancer diagnosis and staging, as well as therapy response monitoring and radiotherapy planning. Finally, the possible impact of these developments on the European Association of Nuclear Medicine
\end{abstract}

Albert J. Arends, Ronald Boellaard, Jorn A. van Dalen, Mark Lubberink, and Antoon T.M. Willemsen have contributed equally to the paper.

Eric P. Visser

eric.visser@radboudumc.nl

1 Department of Radiology and Nuclear Medicine, Radboud University Medical Centre, Nijmegen, The Netherlands

2 MIRA Institute for Biomedical Technology and Technical Medicine, University of Twente, Enschede, The Netherlands

3 Department of Nuclear Medicine, Isala Hospital, Zwolle, The Netherlands

4 Department of Medical Physics, Catharina Hospital, Eindhoven, The Netherlands
(EANM) guidelines and EANM Research Ltd. (EARL) accreditation for FDG-PET/CT tumor imaging is discussed.

Keywords Time-of-flight $\cdot$ Point-spread-function $\cdot$ Digital PET $\cdot$ PET/MR $\cdot$ Lesion detectability $\cdot$ EARL

\section{Introduction}

PET/CT is nowadays widely used in oncology and has become an essential multimodality imaging method that provides both anatomic and metabolic information [1, 2]. PET/ CT imaging is important for the detection, localization, characterization, and staging of cancer [2]. However, the two main limitations of PET are the relatively low spatial resolution, which results in a partial-volume effect (PVE) affecting images both visually and quantitatively [3], and the generally low signal-to-noise ratio (SNR). The PVE limits the detection of small, low-contrast lesions (typically $<2 \mathrm{~cm}$ ), since they appear to be larger while their radiopharmaceutical uptake

5 Department of Nuclear Medicine \& Molecular Imaging, University of Groningen, University Medical Centre Groningen, Groningen, The Netherlands

6 Department of Radiology and Nuclear Medicine, VU University Medical Center, Amsterdam, The Netherlands

7 Department of Medical Physics, Isala, Zwolle, The Netherlands

8 Department of Surgical Sciences, Uppsala University, Uppsala, Sweden

9 Department of Medical Physics, Uppsala University Hospital, Uppsala, Sweden 
appears to be lower than the actual value, due to spill out of activity [4]. In addition, this also decreases the detection sensitivity itself when the signal-to-noise ratio of these lesions becomes too small. These effects are especially important when accurate quantification is needed. In recent years, there have been multiple advances in PET/CT that potentially improve cancer imaging and small lesion detection. In this article, these recent advances in PET/CT technology are explained. Also, the potential consequences of these developments for the EANM guidelines and EARL accreditation for FDG-PET imaging are discussed.

\section{New PET technologies and image reconstruction methods}

In this section, an overview of several PET technological developments that took place during the last decade will be given, as well as a short description of their underlying principles. In particular, this review addresses TOF [5], PSF modeling [6], MAP-based reconstruction [7], smaller voxels [8], respiratory gating [9], metal artefact reduction [10], as well as hardware improvements like CBM [11], the development of solid-state photodetectors using digital photon counting technology [12] and the introduction of combined PET/MR imaging [13].

Our descriptions will be limited to those features that are currently available in commercial, clinical whole-body PET/ CT, and PET/MR systems. Nevertheless, still newer developments are under way, and might enter the market within the coming years. Among these, the most important ones in our opinion, could be the following. New PET reconstruction methods for which PET attenuation correction by $\mathrm{CT}$ is not necessary [14]. This can reduce or avoid several artefacts (motion, metal) in the PET images, and leads to lowering of the radiation dose. Further, a substantial improvement of the TOF timing resolution (see next section) can be expected [5], thus improving image quality, reducing scan time, or reducing administered activity. Finally, scanners with very large axial FOV, such as the total body system proposed by Cherry et al. [15] could provide an even larger improvement of these parameters.

\section{Time-of-flight}

PET imaging is based on the detection of annihilation photons along a line-of-response (LOR). When the difference in arrival time between two annihilation photons is known, the location from which these photons originated can be determined. If this difference equals $\Delta t$, the location of the annihilation event, with respect to the midpoint between the two detectors, is given by $\Delta x=c \Delta t / 2$, where $c$ is the speed of light $\left(3 \times 10^{8} \mathrm{~m} / \mathrm{s}\right)$. This technique is called time-of-flight PET.

In 2006, the first commercial whole-body TOF-PET scanners were introduced. These PET scanners use lutetium oxyorthosilicate (LSO) or lutetium-yttrium oxyorthosilicate (LYSO) scintillators, which provide a timing accuracy of $350-550 \mathrm{ps}$, resulting in a localization accuracy of 5.3$8.3 \mathrm{~cm}$. Table 1 shows vendor-specific timing and localization accuracy information. The spatial resolution of PET without TOF is already in the order of several millimeters. This indicates that TOF information will not directly lead to a higher spatial resolution. However, the incorporation of TOF information in the PET image reconstruction algorithm does provide images with a higher SNR, which improves the detection of small lesions with relatively low activity that would otherwise have been indistinguishable due to background noise. The SNR is approximated by $\mathrm{SNR}_{\mathrm{TOF}} \approx \sqrt{ }(D / \Delta x) \quad \mathrm{SNR}_{\text {non- }}$ TOF where $D$ is the effective patient diameter [25]. Therefore, the effect of TOF is most pronounced in obese patients $[5,25$, 26]. It has been shown that the SNR (as a property of the image) is proportional to the square root of the noise equivalent counts (NEC) [27], which is a property of the PET scanner. The increase in SNR is sometimes regarded as a gain in counts: a TOF image is equivalent to a non-TOF image obtained with a larger number of counts, where $D / \Delta x$ is called the gain factor. The sensitivity times this gain factor is sometimes called the effective sensitivity. In other words, the incorporation of TOF information increases the effective sensitivity. This can be used to provide better image quality and improved lesion detection, or to shorten the scan time while keeping the same image quality with better clinical workflow and added comfort for the patient, or finally to reduce radionuclide costs and reduce radiation dose to the patient and hospital personnel with the same scan time and image quality.

\section{Point-spread-function modeling}

Iterative image reconstruction methods use a system matrix that couples the coincidence counts along each LOR to the activity in the different voxels. In principle, this matrix takes into account all processes that influence the measured counts along each LOR. Among these are resolution degrading effects such as positron range, photon non-colinearity, and detector-related effects, including crystal widths, inter-crystal scattering, and inter-crystal penetration (depth of interaction effects). Resolution modeling or PSF modeling takes into account these effects during image reconstruction [6]. However, PSF modeling can also be applied as a post-reconstruction deconvolution [28]. The first method has been implemented by Siemens (HD) and GE (SharpIR), while the second method is used by Philips, as can be seen in Table 1 .

It has been demonstrated that PSF modeling in PET reconstructions leads to higher and more uniform spatial resolution over the transaxial FOV [29-31]. Special attention should be given to some pitfalls, noise and Gibbs artefacts can be amplified [32]. However, for noise, this depends on its definition. As explained by Alessio et al. [33], PSF modeling can reduce 


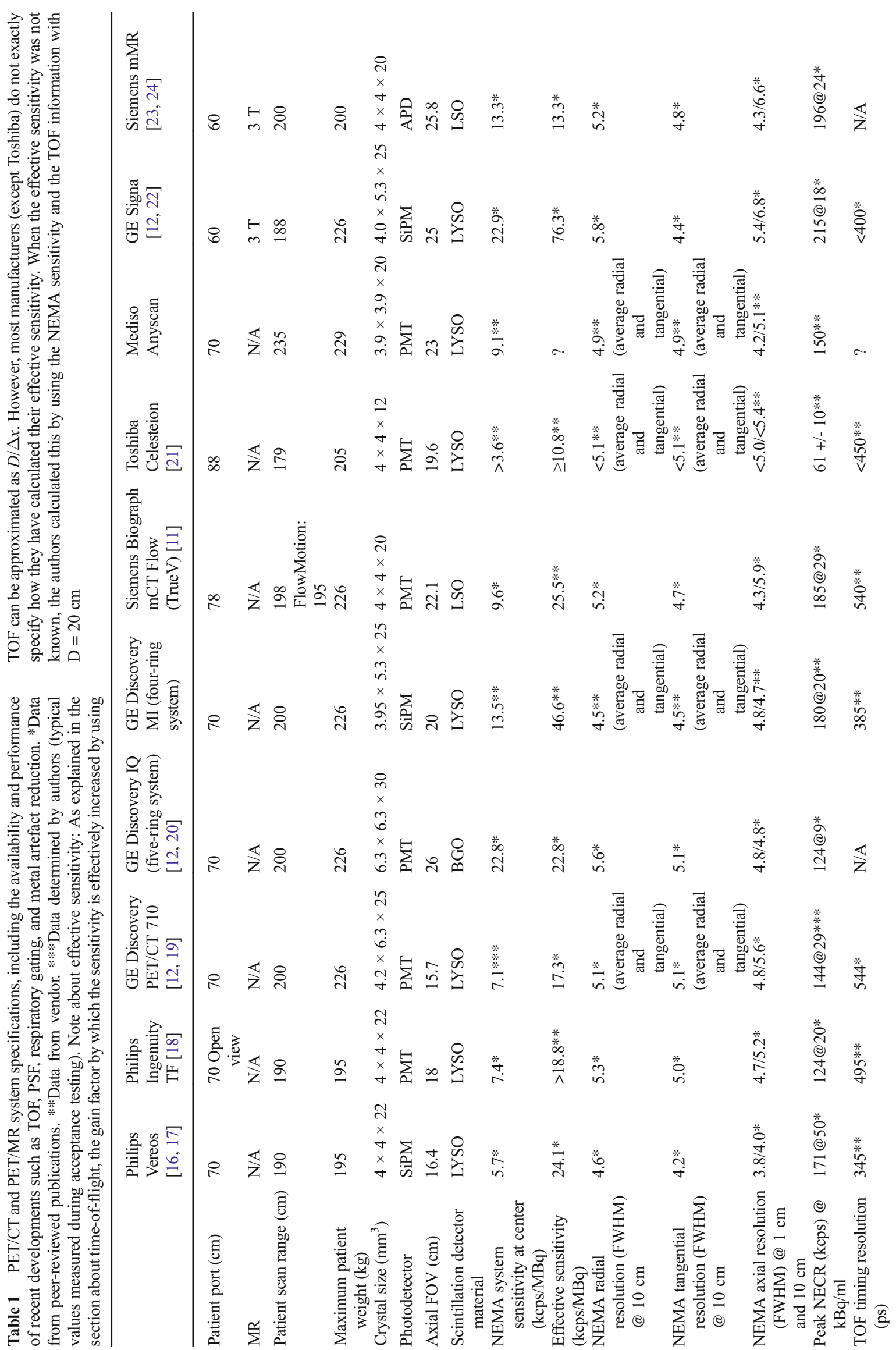


noise when it is defined as intensity variation on a voxel-tovoxel basis, but may increase the ensemble standard deviation of mean lesion uptake. Also, spatially correlated noisy patterns can be introduced, especially for low count statistics [34].

An example of a clinical PET scan demonstrating the impact of TOF and PSF is shown in Fig. 1. It is interesting to note that although PSF modeling was developed and tested mainly for ${ }^{18} \mathrm{~F}-\mathrm{FDG}$ imaging, it clearly also enhances small lesion detectability using ${ }^{68} \mathrm{Ga}$-based tracers. Apparently, this is not hampered by the higher positron energy and larger range for ${ }^{68} \mathrm{Ga}$ versus ${ }^{18} \mathrm{~F}$.

\section{Bayesian penalized likelihood}

When using conventional iterative reconstruction algorithms based on maximum likelihood estimation maximization (MLEM) such as ordered subset expectation maximization (OSEM), the quantitative accuracy of the resulting images improves (the standardized uptake values (SUVs) of lesions increase) when the number of iterations is increased. However, image noise levels also increase with each iteration, hampering visual small lesion detection. As a compromise, some bias (underestimation of SUV in smaller lesions) is allowed in the reconstructed images in return for reduced noise levels, by stopping the iterative process after a limited number of iterations, or by applying post reconstruction spatial smoothing [35].

Bayesian methods are applied in PET image reconstructions to further improve the quality of reconstructed images by taking advantage of prior knowledge of the image, e.g., non-negativity of the tracer concentration, limited variation between neighboring voxels (while preserving real edges), or anatomical information for example from CT. The Bayesian penalized likelihood technique (BPL) or MAP algorithm (for instance as incorporated in Q.Clear (GE) [7]) allows effective convergence of image accuracy while suppressing noise, by using a penalty function $[7,36]$. With every iteration, the outcomes with lower variation between neighboring voxels are slightly favored over noisier ones. The strength of this penalty term is chosen to match the procedure type. A substantial number of iterations (typically 25) warrants convergence without amplifying noise, resulting in improved image quality and increased SUV, particularly in small lesions when compared with reconstruction techniques without using MAP [7, 35, 37]. An example is given in Fig. 2.

\section{Small voxel reconstruction}

In current practice, the image voxel size for whole-body FDGPET scans is typically around $4 \times 4 \times 4 \mathrm{~mm}^{3}[18,38,39]$, which is in the order of the NEMA spatial resolution of the PET scanner [40], defined as the full width at half and tenth maximum (FWHM/FWTM) of a point source when 
reconstructed using filtered back-projection without any corrections. Recent studies demonstrated that the use of smaller voxels and corresponding larger matrices, in combination with TOF-PET/CT systems, improves the detection of small lesions [8, 41-43]. Li et al. [41] demonstrated that using a $400 \times 400$ matrix $\left(2 \times 2 \mathrm{~mm}^{2}\right)$ resulted in more detected lymph nodes and a better visual image quality, as compared to a $200 \times 200$ matrix $\left(4.1 \times 4.1 \mathrm{~mm}^{2}\right)$. Furthermore, Koopman et al. [8] showed that the use of $2 \times 2 \times 2 \mathrm{~mm}^{3}$ instead of $4 \times 4 \times 4$ $\mathrm{mm}^{3}$ voxels was preferred by physicians, based on rankings including lesion sharpness, lesion contrast, and diagnostic confidence. Moreover, the use of $2 \times 2 \times 2 \mathrm{~mm}^{3}$ voxels resulted in an increase in $\mathrm{SUV}_{\text {mean }}, \mathrm{SUV}_{\text {max }}$, and SNR for small lesions $(<11 \mathrm{~mm})$ in patients. This is also demonstrated in Fig. 3. Additionally, they found that the contrast recovery coefficients (as defined in their paper) for phantom spheres were more accurate using $2 \times 2 \times 2 \mathrm{~mm}^{3}$ voxels [8]

A drawback of the use of small voxels is an increase of noise in the PET images as smaller voxels imply fewer counts per voxel [8]. These higher noise levels may result in more false-positive findings [44].

\section{Respiratory gating}

Respiratory motion causes blurring of lesions in the thorax and upper abdomen, and can cause additional artefacts because of an inaccurate attenuation correction due to a mismatch between PET and CT [45]. This results in a lower detectability of tumors, inaccurate SUVs, and sub-optimal radiotherapy treatment planning $[46,47]$. Respiratory gating can be used to create an essentially motion-free PET image. There are two methods that are most common. For the first method, the respiration of the patient is tracked and only a part of the PET data is used to reconstruct a motion-free image. For the second method, the respiration is also tracked, but all PET data is used to reconstruct a motion-free image by translating gated images of the different respiratory phases. In recent years, several respiratory gating methods have been developed for PET imaging $[46,48]$. For the first method, to maintain image quality, respiratory gating requires a longer scan time and/or a higher injected activity. Therefore, respiratory gating is nowadays not routinely used for diagnostic imaging $[49,50]$. However, it is more commonly applied for radiotherapy planning, where an accurate delineation and quantification is even more important [51-53].

Different vendors offer different respiratory gating methods. Philips, GE, and Toshiba use a phase-based gating method [54]. Siemens also allows phase-based gating, but in addition offers an amplitude-based optimal gating method, called HD $\cdot$ Chest. With this method only PET data collected from the respiratory amplitude range with the least amount of motion are used [9, 46]. GE also introduced Q.Freeze, which should only be used for diagnostic purposes. Q.Freeze is a phase-based gating method in combination with a non-rigid translation of the other phases, so all collected data are used for the final motion free image [48]. An example of the impact of respiratory gating on a PET image is shown in Fig. 4.

\section{Metal artefact reduction}

Metal artefact reduction is a standard tool in stand-alone CT systems and different methods are well described in the literature [55]. However in PET/CT, reduction of metal artefacts is relatively new, not commonly implemented, and little research has been performed on the impact of CT metal artefacts on PET imaging. Artefacts on CT images can influence the PET reconstruction, as CT data are used for PET attenuation correction. If the region of interest is located near the implant, the metal not only distorts the CT image but also influences the quantification of radiotracer uptake and can reduce the image quality and interpreter confidence $[10,56]$. Metal artefact reduction is important for diagnosis [57] and therapy planning [58] in head and neck cancer, and it can improve the image quality of ${ }^{68} \mathrm{Ga}$-PSMA PET studies for metastasis detection in patients with one or two hip prostheses [10,59].

Recently, iterative metal artefact reduction was introduced for some PET/CT scanners. Siemens introduced the iMAR algorithm [10], Philips introduced O-MAR and Toshiba SEMAR. It is expected that these algorithms result in an improved quantification and interpretation of the PET image near metal implants. An example is shown in Fig. 5.

\section{Continuous bed motion}

Due to the limited axial FOV of PET scanners, more than one bed position is generally needed to cover the section of the body that needs to be imaged. Since the sensitivity decreases toward the edges of the axial FOV, these bed positions are chosen to partly overlap to improve the uniformity in sensitivity along the axial direction [60]. Recently, CBM acquisition was introduced by Siemens (FlowMotion). The PET scanner shows similar performance compared to its predecessor system with discrete bed positions. The image quality was also similar for both techniques, with the exception of slightly increased noise levels for the planes at the edges of the outer bed positions in the standard acquisition [11, 61].

However, an advantage of the CBM technology is that the scan range can be selected without being restricted to a discrete number of bed positions, thus on average saving scan time by using a shorter scan range [62]. CBM could result in less CT radiation exposure due to this shorter range [62]. Finally, it has been stated that patients prefer the more fluent scanning of the CBM method over the more abrupt movements using discrete bed positions $[61,62]$. 
a

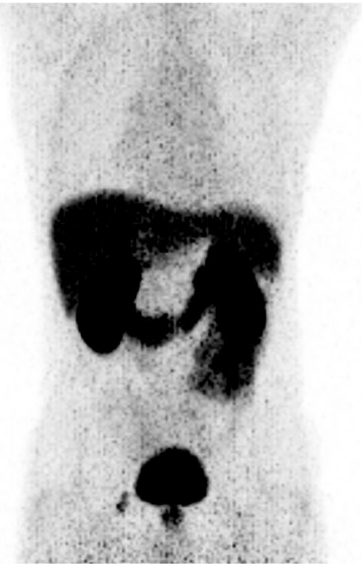

b

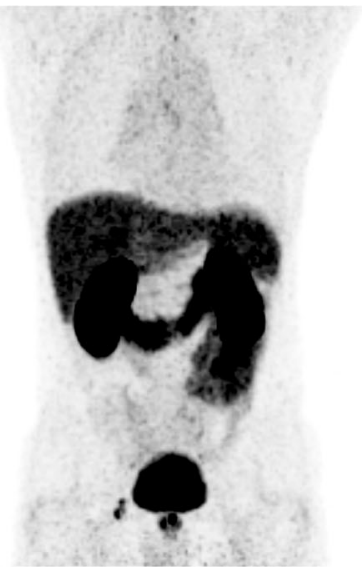

C

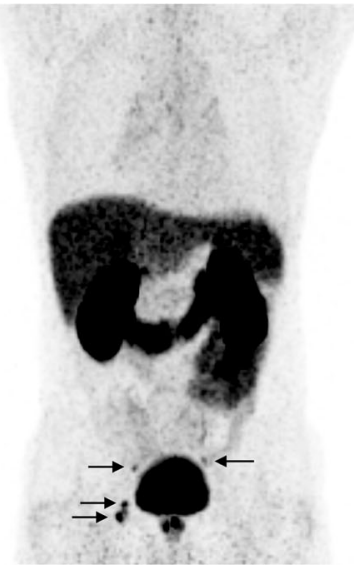

Fig. $1{ }^{68}$ Ga-labeled prostate-specific membrane antigen (PSMA) maximum intensity projection PET images (mCT, Siemens) of a patient with metastasized prostate cancer. PSMA uptake is visible in the prostate and four metastases (two lesions in the acetabulum (right), and two parailiac lymph nodes (left and right)). All images were reconstructed with a transaxial matrix size of $256 \times 256$, pixel size of $3.1 \times 3.1 \mathrm{~mm}^{2}$. (a) PET reconstruction without PSF modeling and without TOF, (b) PET reconstruction with PSF modeling and without TOF, and (c) a PET reconstruction with both PSF modeling and TOF (data are from Radboudumc, Nijmegen, The Netherlands)

\section{Solid-state and digital PET}

Recently, three vendors introduced PET scanners based on solid-state photodetectors, replacing the conventional photomultiplier tubes (PMTs). Siemens introduced their mMR PET/MR scanner that uses avalanche photodiodes (APD), which can operate in a magnetic field, thus offering the possibility of constructing an integrated PET/MR scanner. a

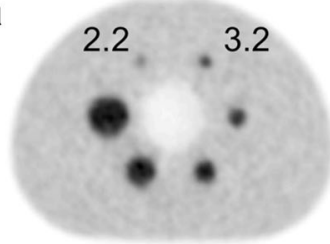

OSEM

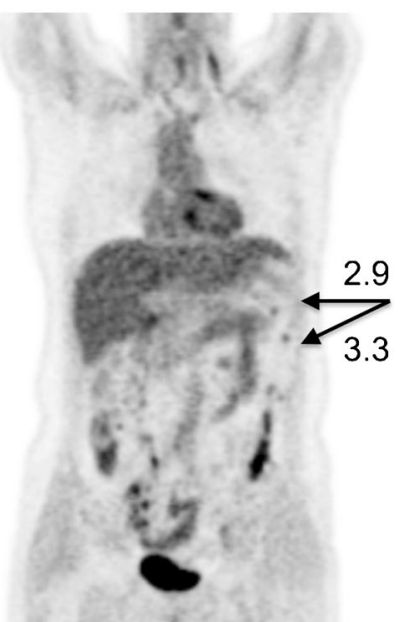

b

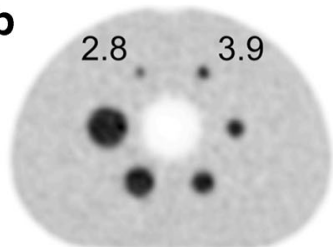

TOF-OSEM + PSF

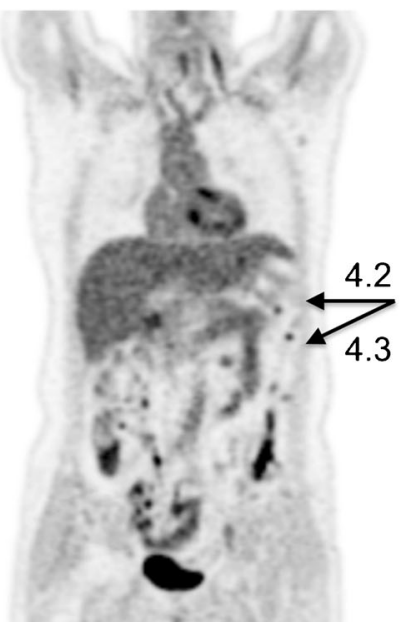

C

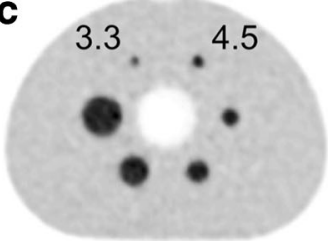

BSREM + PSF

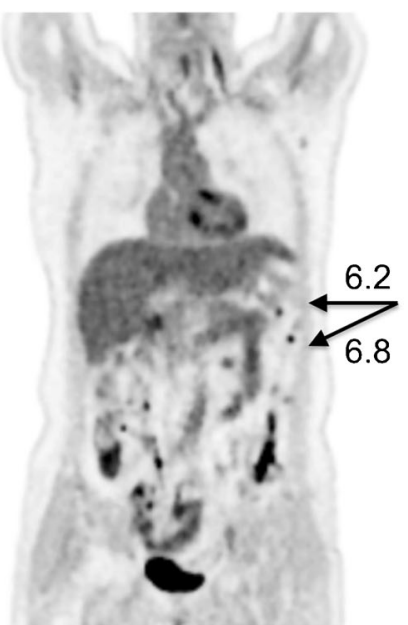

Fig. 2 Top row: images of a 100 Mcounts acquisition of the NEMA image quality phantom (sphere-to-background activity concentration ratio 4:1). Measured sphere-to-background ratios (hottest pixel) are given for the two smallest spheres. Bottom row: ${ }^{18} \mathrm{~F}-\mathrm{FDG}$ PET images (four-ring Discovery MI, GE) of a patient with ovarian cancer with peritoneal carcinomatosis, (a) reconstructed using OSEM, (b) TOFOSEM with PSF modeling, and (c) block-sequential regularized expectation maximization (BSREM; Q.clear) with PSF modeling and a beta-value of $400 . \mathrm{SUV}_{\max }\left[\mathrm{g} / \mathrm{cm}^{3}\right]$ is given for the two lesions. Note the much better recovery in the small lesions when adding TOF and PSF, with further improvement for BSREM, optimized for BPL. The beta value in the BSREM reconstruction was chosen to result in similar background variability in the BSREM and TOF-OSEM images of the NEMA phantom (data are from Uppsala University Hospital, Uppsala, Sweden) 
a
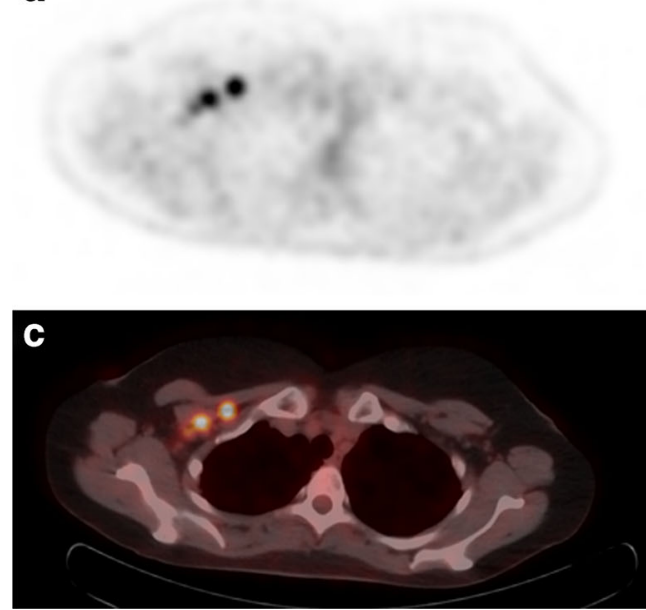

Fig. $3{ }^{18}$ F-FDG PET/CT images (Ingenuity TF, Philips) of a patient with metastasized breast cancer. The reconstructions were made without PSF modeling, but with TOF. (a, c) A standard $4 \times 4 \times 4 \mathrm{~mm}^{3}$ voxel reconstruction and $(\mathbf{b}, \mathbf{d})$ a small $2 \times 2 \times 2 \mathrm{~mm}^{3}$ voxel reconstruction.

GE introduced their Signa PET/MR scanner using silicon photomultipliers (SiPM), which can also operate in a magnetic field. Philips introduced the Vereos PET/CT scanner based on SiPMs with digital readout, and GE released their Discovery MI PET/CT scanner, also based on SiPMs with digital readout.

In case of the digital PET scanner from Philips, the digital SiPMs are capable of detecting and processing single scintillation photons because their elements match the size of the scintillator crystal elements and they incorporate electronics to achieve a one-to-one relation between the scintillator crystal

\section{a}

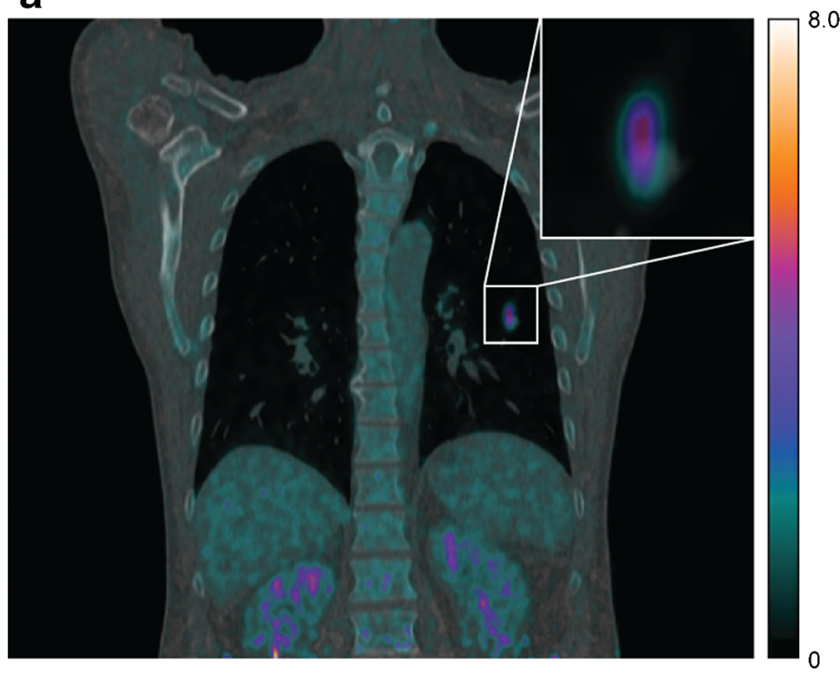

Fig. $4{ }^{18} \mathrm{~F}-\mathrm{FDG}$ PET/CT images (mCT, Siemens) of a patient with a non-small cell lung cancer lesion in the left lower lobe. (a) Non-gated and (b) an essentially motion-free image (HD $\cdot$ Chest). Both PET images have been reconstructed with a matrix size of $400 \times 400$, pixel size of $2 \times 2 \mathrm{~mm}^{2}$, with PSF modeling and TOF. For the non-gated images, the b
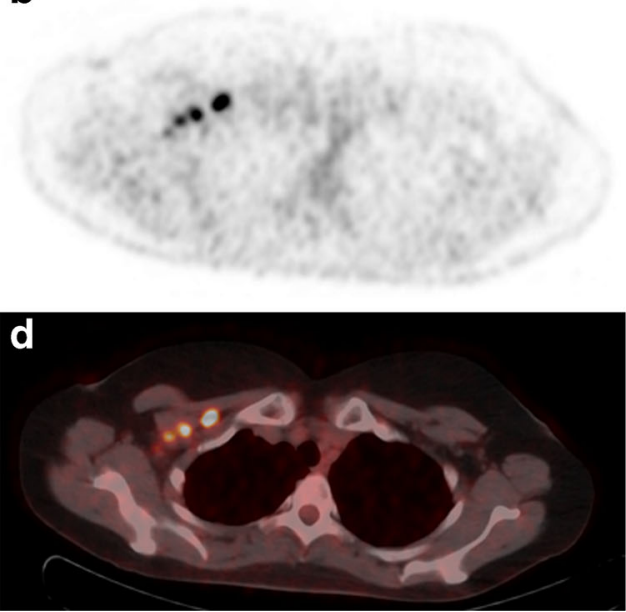

On the small-voxel images, there is an improved visualization of axillary lymph nodes, with an increase of $\mathrm{SUV}_{\max }$ of more than $65 \%$ for the small lymph nodes (data are from Isala Hospital, Zwolle, The Netherlands)

elements and the digital photomultipliers [63-65]. In terms of system performance, this design results in an improved spatial and timing resolution and relatively high maximum count rates. In case of the Discovery MI scanner (GE), 12 crystals $(4 \times 3)$ are coupled to an array of SiPMs $(3 \times 2)$, much like the block design of analogue PMT-based scanners. This reduces count-rate capability and spatial resolution compared to oneto-one coupling of crystals and SiPMs, but improves sensitivity.

Based on phantom and patient studies that were recently performed on a digital PET system [16, 66, 67], it is expected

\section{b}

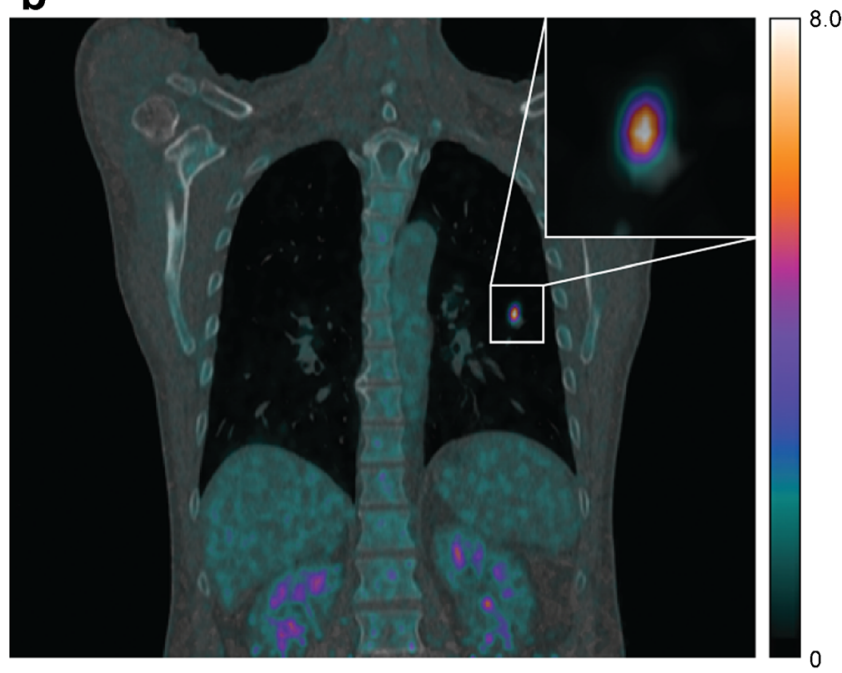

first $35 \%$ (126 s) of the acquired data was used for image reconstruction, resulting in an equal number of acquired true coincidences as the gated image. There is a considerable increase in $\mathrm{SUV}_{\text {mean }}$ of $70 \%$ and a decrease in volume of $80 \%$. Images have been reproduced from [46] 


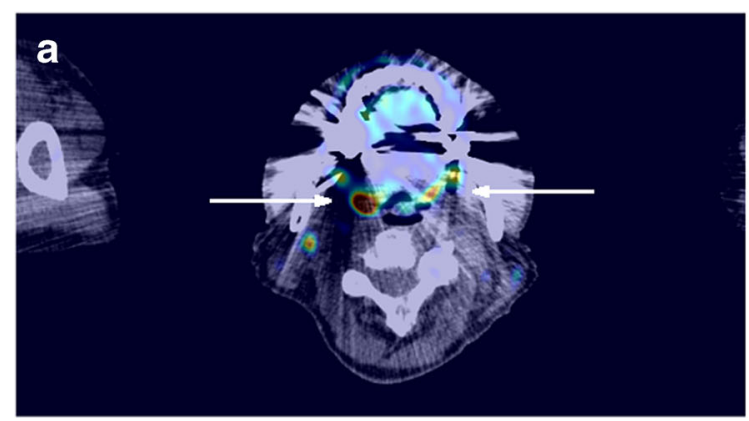

Fig. $5{ }^{18} \mathrm{~F}-\mathrm{FDG}$ PET/CT images (mCT, Siemens) of a patient with uptake in the palatine tonsils (arrows in a), and ${ }^{18} \mathrm{~F}-\mathrm{FDG}$-avid lymph nodes (arrows in b). Both PET images have been reconstructed with a matrix size of $200 \times 200$, pixel size of $4 \times 4 \mathrm{~mm}^{2}$, with PSF modeling and TOF. The metal artefact is visible on the (a) standard PET/CT

that digital PET can provide a higher image quality and/or allow for a lower radiopharmaceutical dose and improved small lesion detection for oncology scans, as compared to an analogue PET system with PMTs. Figure 6 shows PET images of an analogue, PMT-based system and a digital PET system, of a NEMA image quality phantom (sphere diameters 10 $37 \mathrm{~mm}$ ) and a micro hollow sphere phantom (sphere diameters 4-8 $\mathrm{mm}$ ). The reconstructed images demonstrate that image quality and small object detection improve using reconstruction settings with small voxels, on both the analogue and the digital PET. Furthermore, there is a higher contrast of the smallest spheres on the digital PET images as compared to the analogue PMT-based PET. Nguyen et al. [68] reported their initial experience in cancer patients with a prototype digital PET scanner compared to an analogue PET system

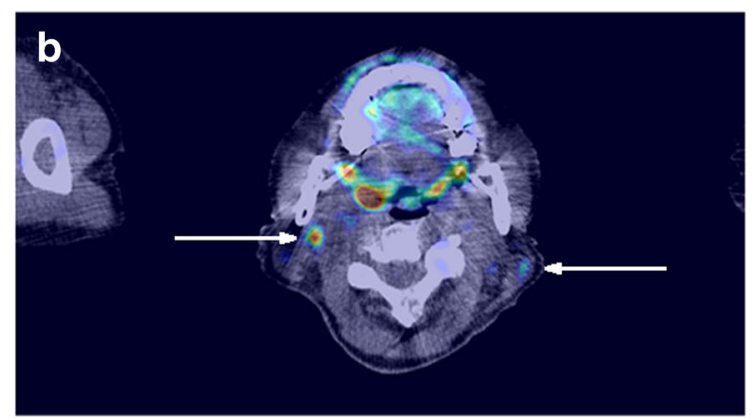

reconstruction, while the (b) $\mathrm{PET} / \mathrm{CT}$ reconstruction with metal artefact reduction (iMAR) shows fewer CT artefacts. There is an $\mathrm{SUV}_{\text {mean }}$ increase from 2.5 to $2.8 \mathrm{~g} / \mathrm{cm}^{3}$ when iMAR is used for the tonsil. Images have been reproduced from [10]

with PMTs. They found a better image quality, diagnostic confidence, and accuracy with their digital PET.

\section{Hybrid PET/MR imaging}

During the development of hybrid PET/MR systems, two major challenges needed to be overcome. First of all, conventional PET photodetectors are based on PMTs that cannot be operated in the high magnetic field of an MR scanner and are too large to allow placement inside an MR body coil whilst still leaving a sufficiently large patient opening. Integrated PET/ MR was achieved using (analogue) APDs or SiPMs for conversion of the light produced by the scintillator crystals. In addition to their ability to function properly in a magnetic field, both APDs and SiPMs are much smaller than traditional a
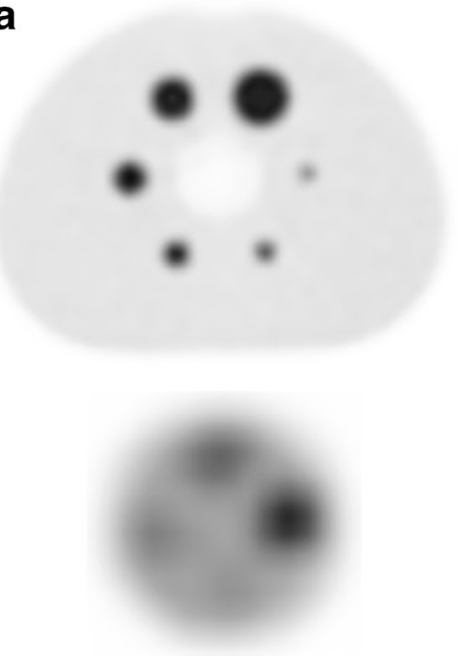

b
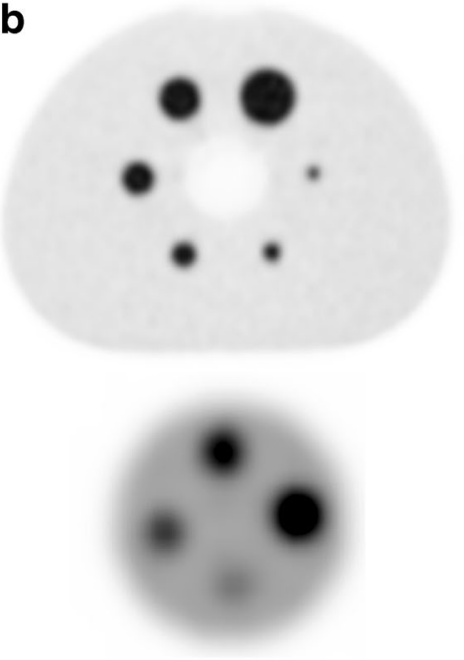
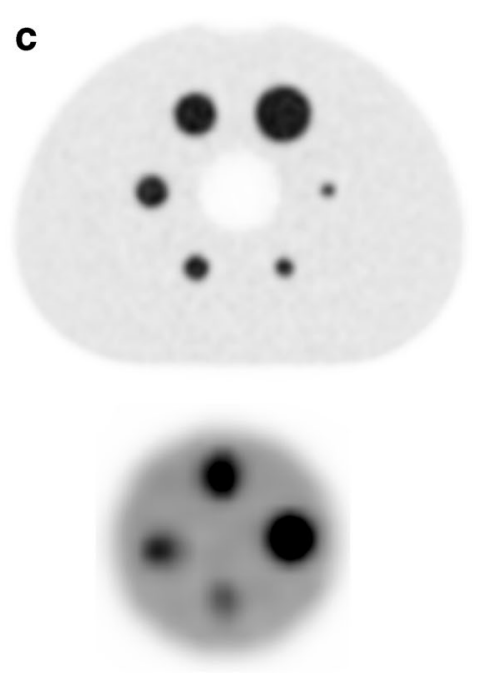

Fig. 6 PET images of a NEMA phantom (sphere diameters 10-37 mm) and micro phantom (sphere diameters $4-8 \mathrm{~mm}$ ), filled with 20 and $2 \mathrm{kBq} /$ $\mathrm{ml} \mathrm{FDG}$ in the spheres and the background, respectively. Data were acquired on an analogue, PMT-based PET (Ingenuity TF, Philips) and a digital SiPM-based PET (Vereos, Philips). (a) Images of the analogue
PET that fulfils EARL requirements. (b) Images of the analogue PET using $2 \times 2 \times 2 \mathrm{~mm}^{3}$ voxel reconstruction. (c) Images of a digital PET using a $2 \times 2 \times 2 \mathrm{~mm}^{3}$ voxel reconstruction (data are from Isala Hospital, Zwolle, The Netherlands) 
PMTs, allowing for detector rings of about $5 \mathrm{~cm}$ thickness inside a $70-\mathrm{cm}$ MR bore, leaving a $60-\mathrm{cm}$ patient port diameter. An advantage of SiPMs compared to APDs is that SiPMs allow for TOF, whereas APDs, due to their timing resolution of about 2000 ps, do not. Specifications of the PET components for two fully integrated PET/MR systems are given in Table 1.

The second major challenge of quantitative PET with PET/ MR, is that the PET attenuation correction needs to be derived from MR images, which essentially provide proton density rather than attenuation coefficients. Most PET/MR systems employ a dedicated (fast) MR sequence, followed by segmentations or tissue classification of the resulting MR image and assigning a priori known attenuation coefficients to a limited number of segmentation or tissue classes. This approach has several limitations. First of all, bone tissue is typically not included in this process and its attenuation is assumed to be equivalent to soft tissue attenuation. Secondly, lungs are segmented and assigned a uniform attenuation coefficient. Thirdly, the patient couch, fixation devices, and the coils used for MR image acquisition are not detected by the MR scanner and dedicated predefined attenuation templates need to be added to the attenuation image to compensate for them. Fourthly, the MR FOV is typically smaller than that of the PET scanner and truncation of the MR image in the transaxial direction is often observed, resulting in incomplete attenuation coefficient images and thus incorrect attenuation correction of the PET data. For most of the limitations indicated above, solutions have been proposed but not all of them are yet routinely available on all systems. For example ultra-short echo time (UTE) or zero echo time (ZTE) MR can be used to visualize bone and has only recently been introduced for brain PET/MR [69]. Another approach would be the use of CTbased templates which are registered onto the patients MR images and finally combined and processed to generate patient-specific attenuation images [69]. MR truncation artefacts in the attenuation images can be solved by first performing a PET reconstruction without attenuation, then derive the outer contour of the patient from this image and assign soft tissue attenuation to the tissues missed in the MR image [69]. However, advanced reconstruction methods, such as maximum likelihood of activity and attenuation (MLAA), might also be used to correct for MR truncation or otherwise incorrect attenuation maps [70-72]. A more complete overview of current PET/MR technologies, opportunities and challenges can be found in a review by Quick and Boellaard [73].

\section{Possible future implications of technological developments on imaging guidelines and applications}

To date, most of the new technologies that were discussed in this paper are not yet widely spread in clinical practice. However, several of these, such as digital photodetector technology, PET/MR and novel PET reconstruction methods will become more available. We expect that they will be increasingly clinically used in the next decade and will have a large impact on image quality, lesion detection, and quantification in cancer PET imaging. These new technological developments thus provide a technology push for the evolution of new standards and imaging guidelines.

\section{Imaging guidelines and quantitative standards}

The EANM guidelines for FDG-PET/CT tumor imaging and the associated PET/CT system accreditation program run by EARL aim to harmonize the use of FDG-PET/CT in oncology as a quantitative imaging biomarker in multicenter studies [39]. To date, the EANM/EARL standard is based on the technological status for the majority of the installed PET/CT systems. In order to allow sites to benefit from the advantages of the new technologies described, two different PET reconstructions could be made: one optimized for visual interpretation and another meeting international quantitative standards $[39,74-76]$. With the introduction of new acquisition and reconstruction techniques in the latest scanners from multiple vendors, and assuming that the availability and presence of PET scanners using older technology will decrease, it is expected that these technologies will become widely spread during the next 5 to 10 years. Consequently, EARL standards will need to be updated over time and the implication of new technologies on harmonized quantitative performance is presently being explored by EARL as discussed in more detail elsewhere in this supplement issue [77].

\section{New applications facilitated by new technologies}

The improved image quality can be used to adjust administered activity and/or scan duration. In 2013, de Groot et al. [78] published an optimized FDG-activity regimen, which is based on a quadratic relation between FDG-activity and patient's body weight. They demonstrated that when using a quadratic administration regimen, the image quality (in terms of SNR in the liver) remains constant for patients with various body masses. This FDG-activity regimen has been mentioned as an alternative to the linear regimen in the second version of the EANM guidelines for FDG-PET tumor imaging [39]. Recently, a technical note was published by Koopman et al. [79] describing how to derive an FDG-activity formula, taking into account both EANM guidelines [39, 80] and a quadratic relation between FDG-activity and patient's body weight. Their equation can be applied for all PET/CT systems, regardless of their technological status. A drawback of the quadratic administration of FDG-activity is that it requires a high amount of FDG-activity in obese patients. Alternatively, a quadratic-dependent duration of the PET scan could be implemented in these cases. 
An example of a new application of PET/CT that has been facilitated by the recent developments in PET/CT technology is the use of ${ }^{90} \mathrm{Y}-\mathrm{PET} / \mathrm{CT}$ imaging in patients with liver metastasis who were treated by selective internal radiation therapy (SIRT). ${ }^{90} \mathrm{Y}$ is a radionuclide with a very small positron fraction $\left(31.9 \times 10^{-6}\right)$ and therefore it is challenging to use it for PET imaging [81]. However, several studies have recently compared Bremstrahlung ${ }^{90} \mathrm{Y}$-single-photon emission computed tomography (SPECT)/CT with ${ }^{90} \mathrm{Y}-\mathrm{PET} / \mathrm{CT}$ and demonstrated that ${ }^{90} \mathrm{Y}-\mathrm{PET} / \mathrm{CT}$ scans using state-of-the-art TOFPET systems provide a higher image quality with improved lesion detection and more accurate quantification and dosimetry [82-86].

Furthermore, the recent developments in PET/CT technology facilitate the use of low-count-rate PET studies such as imaging with ${ }^{124} \mathrm{I}$, which is performed in the follow-up of thyroid cancer. In general, the image quality for the ${ }^{124} \mathrm{I}-$ PET scan is poor due to the complex decay scheme and especially the emission of prompt gamma rays with an energy of $602.7 \mathrm{keV}$, well within the standard energy window of a PET scanner. Furthermore, even higher energy gammas are present, which can downscatter into the energy window, or increase the dead time. For such a radionuclide, TOF results in a better SNR for the same number of counts [5, 87]. It is expected that recent developments in PET/CT technology, combined with a careful application of correction methods for the prompt gammas [88], further facilitate the use of ${ }^{124}$ I-PET/ CT [89] (or tracers labeled with other radionuclides such as ${ }^{89} \mathrm{Zr}[90]$ ) with an improved image quality and a more accurate quantification [91].

\section{Conclusions}

In recent years, the development of PET/CT scanners has mainly focused on improved small lesion detection. The introduction of TOF, PSF modeling, and smaller voxels were the main reasons for this improvement. Also, an increased axial length increased the sensitivity of the scanner [60], while the spatial resolution was improved by reducing the size of the scintillator crystal element and by using smaller voxels [60]. Other reconstruction techniques have been developed for specific problems, such as respiratory gating and metal artefact reduction. Together, all these advancements made it possible to improve the quality and quantification of PET/CT images and optimize radiation dose and scan time.

The increase in effective sensitivity and improved spatial resolution led to an improved visibility of small lesions, which is not only important for detection of lesions and metastases in ${ }^{18} \mathrm{~F}-\mathrm{FDG}-\mathrm{PET} / \mathrm{CT}$ scans, but also for other tracers, for instance the use of ${ }^{68} \mathrm{Ga}$-PSMA for the detection of (lymph node) metastases in patients with prostate cancer, ${ }^{89} \mathrm{Zr}-\mathrm{MAb}$ immunoPET studies, ${ }^{90} \mathrm{Y}$ imaging for patients who are treated for liver metastasis, or ${ }^{124} \mathrm{I}$ imaging for follow-up of thyroid cancer. This implies that PET/CT is nowadays not only used for detection and identification of lesions but has also been increasingly implemented for radiotherapy planning and therapy response monitoring [39]. For these applications, an accurate quantification and repeatability/reproducibility is of the utmost importance. The ongoing improvements discussed in this paper can contribute to this.

Compliance with ethical standards Charlotte van der Vos and Eric Visser received a research grant from Siemens, and Isala has a research cooperation with Philips. Mark Lubberink has received speaking fees and research support from GE Healthcare. The other authors declare that they have no conflicts of interest. This article does not contain any studies with human participants or animals performed by any of the authors. Since this study does not contain any human participants, no informed consent was signed.

Open Access This article is distributed under the terms of the Creative Commons Attribution 4.0 International License (http:// creativecommons.org/licenses/by/4.0/), which permits unrestricted use, distribution, and reproduction in any medium, provided you give appropriate credit to the original author(s) and the source, provide a link to the Creative Commons license, and indicate if changes were made.

\section{References}

1. Schoder H, Erdi YE, Larson SM, Yeung HW. PET/CT: a new imaging technology in nuclear medicine. Eur J Nucl Med Mol Imaging. 2003;30(10):1419-37. doi:10.1007/s00259-003-1299-6.

2. Townsend DW. Dual-modality imaging: combining anatomy and function. J Nucl Med. 2008;49(6):938-55. doi:10.2967/jnumed. 108.051276

3. Takamochi K, Yoshida J, Murakami K, Niho S, Ishii G, Nishimura $\mathrm{M}$, et al. Pitfalls in lymph node staging with positron emission tomography in non-small cell lung cancer patients. Lung Cancer. 2005;47(2):235-42. doi:10.1016/j.lungcan.2004.08.004.

4. Soret M, Bacharach SL, Buvat I. Partial-volume effect in PET tumor imaging. J Nucl Med. 2007;48(6):932-45. doi:10.2967/ jnumed.106.035774.

5. Vandenberghe S, Mikhaylova E, D'Hoe E, Mollet P, Karp JS. Recent developments in time-of-flight PET. EJNMMI Phys. 2016;3(1):3. doi:10.1186/s40658-016-0138-3.

6. Panin VY, Kehren F, Michel C, Casey M. Fully 3-D PET reconstruction with system matrix derived from point source measurements. IEEE Trans Med Imaging. 2006;25(7):907-21. doi:10.1109/ TMI.2006.876171.

7. Teoh EJ, McGowan DR, Macpherson RE, Bradley KM, Gleeson FV. Phantom and clinical evaluation of the Bayesian penalized likelihood reconstruction algorithm Q.Clear on an LYSO PET/CT system. J Nucl Med. 2015;56(9):1447-52. doi:10.2967/jnumed. 115.159301

8. Koopman D, van Dalen JA, Lagerweij MC, Arkies H, de Boer J, Oostdijk AH, et al. Improving the detection of small lesions using a state-of-the-art time-of-flight PET/CT system and small voxel reconstructions. J Nucl Med Technol. 2015;43(1):21-7. doi:10.2967/ jnmt.114.147215.

9. van Elmpt W, Hamill J, Jones J, De Ruysscher D, Lambin P, Ollers M. Optimal gating compared to 3D and 4D PET reconstruction for characterization of lung tumours. Eur J Nucl Med Mol Imaging. 2011;38(5):843-55. doi:10.1007/s00259-010-1716-6. 
10. van der Vos CS, Arens AIJ, Hamill JJ, Hofman C, Panin VY, Meeuwis AP et al. Metal artifact reduction of CT scans to improve PET/CT. J Nucl Med. 2017. doi:10.2967/jnumed.117.191171.

11. Rausch I, Cal-Gonzalez J, Dapra D, Gallowitsch HJ, Lind P, Beyer $\mathrm{T}$, et al. Performance evaluation of the Biograph $\mathrm{mCT}$ flow PET/CT system according to the NEMA NU2-2012 standard. EJNMMI Phys. 2015;2(1):26. doi:10.1186/s40658-015-0132-1.

12. Slomka PJ, Pan T, Germano G. Recent advances and future progress in PET instrumentation. Semin Nucl Med. 2016;46(1):5-19. doi:10.1053/j.semnuclmed.2015.09.006.

13. Wehrl HF, Sauter AW, Divine MR, Pichler BJ. Combined PET/MR: a technology becomes mature. J Nucl Med. 2015;56(2):165-8. doi: 10.2967/jnumed.114.150318.

14. Berker Y, Li Y. Attenuation correction in emission tomography using the emission data-a review. Med Phys. 2016;43(2):807-32. doi:10.1118/1.4938264.

15. Cherry SR, Badawi RD, Karp JS, Moses WW, Price P, Jones T. Total-body imaging: transforming the role of positron emission tomography. Sci Trans Med. 2017;9(381) doi:10.1126/scitranslmed. aaf6169.

16. Miller M, Zhang J, Binzel K, Griesmer J, Laurence T, Narayanan $\mathrm{M}$, et al. Characterization of the Vereos digital photon counting PET system. J Nucl Med. 2015;56(supplement 3):434.

17. Nguyen NC, Vercher-Conejero JL, Sattar A, Miller MA, Maniawski PJ, Jordan DW, et al. Image quality and diagnostic performance of a digital PET prototype in patients with oncologic diseases: initial experience and comparison with analog PET. J Nucl Med. 2015;56(9):1378-85. doi:10.2967/jnumed.114.148338.

18. Kolthammer JA, Su K-H, Grover A, Narayanan M, Jordan DW, Muzic RF. Performance evaluation of the Ingenuity TF PET/CT scanner with a focus on high count-rate conditions. Phys Med Biol. 2014;59(14):3843-59. doi:10.1088/0031-9155/59/14/3843.

19. Yoon HJ, Jeong YJ, Son HJ, Kang D-Y, Hyun K-Y, Lee M-K. Optimization of the spatial resolution for the GE discovery PET/ CT 710 by using NEMA NU 2-2007 standards. J Korean Phys Soc. 2015;66(2):287-94. doi:10.3938/jkps.66.287.

20. Reynes-Llompart G, Gamez-Cenzano C, Romero-Zayas I, Rodriguez-Bel L, Vercher-Conejero JL, Marti-Climent JM. Performance characteristics of the whole-body discovery IQ PET/ CT system. J Nucl Med. 2017; doi:10.2967/jnumed.116.185561.

21. Burr KC, Wang GCJ, Du H, Mann G, Balakrishnan K, Wang J et al., editors. A new modular and scalable detector for a time-offlight PET scanner. New York: IEEE Nuclear Science Symposium and Medical Imaging Conference Record (NSS/MIC); 2012 Oct. 27 2012-Nov. 32012.

22. Grant AM, Deller TW, Khalighi MM, Maramraju SH, Delso G, Levin CS. NEMA NU 2-2012 performance studies for the SiPMbased ToF-PET component of the GE SIGNA PET/MR system. Med Phys. 2016;43(5):2334-43. doi:10.1118/1.4945416.

23. Karlberg AM, Saether O, Eikenes L, Goa PE. Quantitative comparison of PET performance-Siemens Biograph $\mathrm{mCT}$ and $\mathrm{mMR}$. EJNMMI Phys. 2016;3(1):5. doi:10.1186/s40658-016-0142-7.

24. Delso G, Furst S, Jakoby B, Ladebeck R, Ganter C, Nekolla SG, et al. Performance measurements of the Siemens mMR integrated whole-body PET/MR scanner. J Nucl Med. 2011;52(12):1914-22. doi:10.2967/jnumed.111.092726.

25. Conti M. Focus on time-of-flight PET: the benefits of improved time resolution. Eur J Nucl Med Mol Imaging. 2011;38(6):114757. doi:10.1007/s00259-010-1711-y.

26. Lois C, Jakoby BW, Long MJ, Hubner KF, Barker DW, Casey ME, et al. An assessment of the impact of incorporating time-of-flight information into clinical PET/CT imaging. J Nucl Med. 2010;51(2): 237-45. doi:10.2967/jnumed.109.068098.

27. Strother SC, Casey ME, Hoffman EJ. Measuring PET scanner sensitivity - relating countrates to image signal-to-noise ratios using noise equivalent counts. IEEE Trans Nucl Sci. 1990;37(2):783-8. doi:10.1109/23.106715.

28. Perkins A, Narayanan M, Zhang B, Scheuermann J, Karp J, Shao L. Influence of a post-reconstruction resolution recovery algorithm on quantitation. J Nucl Med. 2013;54(supplement 2):2128.

29. Rogasch JM, Steffen IG, Hofheinz F, Grosser OS, Furth C, Mohnike K, et al. The association of tumor-to-background ratios and SUVmax deviations related to point spread function and time-of-flight F18FDG-PET/CT reconstruction in colorectal liver metastases. EJNMMI Res. 2015;5:31. doi:10.1186/s13550-015-0111-5.

30. Akamatsu G, Ishikawa K, Mitsumoto K, Taniguchi T, Ohya N, Baba S, et al. Improvement in PET/CT image quality with a combination of point-spread function and time-of-flight in relation to reconstruction parameters. J Nucl Med. 2012;53(11):1716-22. doi: 10.2967/jnumed.112.103861.

31. Bellevre D, Blanc Fournier C, Switsers O, Dugue AE, Levy C, Allouache D, et al. Staging the axilla in breast cancer patients with 18F-FDG PET: how small are the metastases that we can detect with new generation clinical PET systems? Eur J Nucl Med Mol Imaging. 2014;41(6):1103-12. doi:10.1007/s00259-014-2689-7.

32. Lee YS, Kim JS, Kim KM, Kang JH, Lim SM, Kim HJ. Performance measurement of PSF modeling reconstruction (true $\mathrm{X})$ on Siemens Biograph TruePoint TrueV PET/CT. Ann Nucl Med. 2014;28(4):340-8. doi:10.1007/s12149-014-0815-z.

33. Alessio AM, Rahmim A, Orton CG. Point/counterpoint. Resolution modeling enhances PET imaging. Med Phys. 2013;40(12):120601. doi:10.1118/1.4821088.

34. Grootjans W, Meeuwis AP, Slump CH, de Geus-Oei LF, Gotthardt $\mathrm{M}$, Visser EP. Performance of 3DOSEM and MAP algorithms for reconstructing low count SPECT acquisitions. Z Med Phys. 2016;26(4):311-22. doi:10.1016/j.zemedi.2015.12.004.

35. Teoh EJ, McGowan DR, Bradley KM, Belcher E, Black E, Gleeson FV. Novel penalised likelihood reconstruction of PET in the assessment of histologically verified small pulmonary nodules. Eur Radiol. 2016;26(2):576-84. doi:10.1007/s00330-015-3832-y.

36. Parvizi N, Franklin JM, McGowan DR, Teoh EJ, Bradley KM, Gleeson FV. Does a novel penalized likelihood reconstruction of 18F-FDG PET-CT improve signal-to-background in colorectal liver metastases? Eur J Radiol. 2015;84(10):1873-8. doi:10.1016/j. ejrad.2015.06.025.

37. Rowley LM, Bradley KM, Boardman P, Hallam A, McGowan DR. Optimization of image reconstruction for $90 \mathrm{Y}$ selective internal radiotherapy on a lutetium yttrium orthosilicate PET/CT system using a Bayesian penalized likelihood reconstruction algorithm. J Nucl Med. 2017;58(4):658-64. doi:10.2967/jnumed.116.176552.

38. Conti M. Focus on time-of-flight PET: the benefits of improved time resolution. Eur J Nucl Med Mol Imaging. 2011;38(6):114757. doi:10.1007/s00259-010-1711-y.

39. Boellaard R, Delgado-Bolton R, Oyen WJ, Giammarile F, Tatsch $\mathrm{K}$, Eschner W, et al. FDG PET/CT: EANM procedure guidelines for tumour imaging: version 2.0. Eur J Nucl Med Mol Imaging. 2015;42(2):328-54. doi:10.1007/s00259-014-2961-x.

40. NEMA. National Electrical Manufacturers Association. NEMA standards publication NU 2-2012. Performance measurements of positron emission tomographs. Rosslyn: National Electrical Manufacturers Association; 2012.

41. Li C-Y, Klohr S, Sadick H, Weiss C, Hoermann K, Schoenberg SO, et al. Effect of time-of-flight technique on the diagnostic performance of $18 \mathrm{~F}-\mathrm{FDG}$ PET/CT for assessment of lymph node metastases in head and neck squamous cell carcinoma. J Nucl Med Technol. 2014;42(3):181-7. doi:10.2967/jnmt.114.141192.

42. Zhang J, Wright C, Binzel K, Siva A, Saif T, Nagar V, et al. High definition (HD) and ultra-high definition (UHD) PET reconstructions improves lesion detectability in digital 18F-FDG PET/CT. J Nucl Med. 2016;57(supplement 2):1980. 
43. Morey AM, Noo F, Kadrmas DJ. Effect of using $2 \mathrm{~mm}$ voxels on observer performance for PET lesion detection. IEEE Trans Nucl Sci. 2016;63(3):1359-66. doi:10.1109/TNS.2016.2518177.

44. Sadick M, Molina F, Frey S, Piniol R, Sadick H, Brade J, et al. Effect of reconstruction parameters in high-definition PET/CT on assessment of lymph node metastases in head and neck squamous cell carcinoma. J Nucl Med Technol. 2013;41(1):19-25. doi:10. 2967/jnmt.112.116806.

45. van der Vos CS, Grootjans W, Osborne DR, Meeuwis AP, Hamill JJ, Acuff S, et al. Improving the spatial alignment in PET/CT using amplitude-based respiration-gated PET and respiration-triggered CT. J Nucl Med. 2015;56(12):1817-22. doi:10.2967/jnumed.115.163055.

46. Grootjans W, de Geus-Oei LF, Meeuwis AP, van der Vos CS, Gotthardt $\mathrm{M}$, Oyen WJ, et al. Amplitude-based optimal respiratory gating in positron emission tomography in patients with primary lung cancer. Eur Radiol. 2014;24(12):3242-50. doi:10.1007/s00330-014-3362-z.

47. Nehmeh SA, Erdi YE. Respiratory motion in positron emission tomography/computed tomography: a review. Semin Nucl Med. 2008;38(3):167-76. doi:10.1053/j.semnuclmed.2008.01.002.

48. Minamimoto R, Mitsumoto T, Miyata Y, Sunaoka F, Morooka M, Okasaki M, et al. Evaluation of a new motion correction algorithm in PET/CT: combining the entire acquired PET data to create a single three-dimensional motion-corrected PET/CT image. Nucl Med Commun. 2016;37(2):162-70. doi:10.1097/MNM. 0000000000000423.

49. Grootjans W, Hermsen R, van der Heijden EH, Schuurbiers-Siebers OC, Visser EP, Oyen WJ, et al. The impact of respiratory gated positron emission tomography on clinical staging and management of patients with lung cancer. Lung Cancer. 2015;90(2):217-23. doi: 10.1016/j.lungcan.2015.09.016.

50. Callahan J, Kron T, Schneider ME, Hicks RJ. A prospective investigation into the clinical impact of 4D-PET/CT in the characterisation of solitary pulmonary nodules. Cancer Imaging. 2014;14:24. doi:10.1186/1470-7330-14-24.

51. Wijsman R, Grootjans W, Troost EG, van der Heijden EH, Visser EP, de Geus-Oei LF, et al. Evaluating the use of optimally respiratory gated $18 \mathrm{~F}-\mathrm{FDG}$-PET in target volume delineation and its influence on radiation doses to the organs at risk in non-small-cell lung cancer patients. Nucl Med Commun. 2016;37(1):66-73. doi: 10.1097/MNM.0000000000000409.

52. Callahan J, Kron T, Siva S, Simoens N, Edgar A, Everitt S, et al. Geographic miss of lung tumours due to respiratory motion: a comparison of 3D vs 4D PET/CT defined target volumes. Radiat Oncol. 2014:9:291. doi:10.1186/s13014-014-0291-6.

53. Guerra L, Meregalli S, Zorz A, Niespolo R, De Ponti E, Elisei F, et al. Comparative evaluation of CT-based and respiratory-gated PET/CTbased planning target volume (PTV) in the definition of radiation treatment planning in lung cancer: preliminary results. Eur J Nucl Med Mol Imaging. 2014;41(4):702-10. doi:10.1007/s00259-013-2594-5.

54. Chirindel A, Adebahr S, Schuster D, Schimek-Jasch T, Schanne $\mathrm{DH}$, Nemer U, et al. Impact of 4D-18FDG-PET/CT imaging on target volume delineation in SBRT patients with central versus peripheral lung tumors. Multi-reader comparative study. Radiother Oncol. 2015;115(3):335-41. doi:10.1016/j.radonc.2015.05.019.

55. Gjesteby L, De Man B, Jin YN, Paganetti H, Verburg J, Giantsoudi D, et al. Metal artifact reduction in CT: where are we after four decades? IEEE Access. 2016;4:5826-49. doi:10.1109/Access.2016.2608621.

56. Kamel EM, Burger C, Buck A, von Schulthess GK, Goerres GW. Impact of metallic dental implants on CT-based attenuation correction in a combined PET/CT scanner. Eur Radiol. 2003;13(4):724-8. doi:10.1007/s00330-002-1564-2.

57. Shimamoto H, Kakimoto N, Fujino K, Hamada S, Shimosegawa E, Murakami S, et al. Metallic artifacts caused by dental metal prostheses on PET images: a PET/CT phantom study using different PET/CT scanners. Ann Nucl Med. 2009;23(5):443-9. doi:10.1007/ s12149-009-0254-4.
58. Awan MJ, Siddiqui F, Schwartz D, Yuan J, Machtay M, Yao M. Application of positron emission tomography/computed tomography in radiation treatment planning for head and neck cancers. World J Radiol. 2015;7(11):382-93. doi:10.4329/wjr.v7.i11.382.

59. Abdoli M, Dierckx RA, Zaidi H. Metal artifact reduction strategies for improved attenuation correction in hybrid PET/CT imaging. Med Phys. 2012;39(6):3343-60. doi:10.1118/1.4709599.

60. Cherry SR, Sorenson JA, Phelps ME. Physics in nuclear medicine. 4th ed. Philadelphia: Elsevier/Saunders; 2012.

61. Schatka I, Weiberg D, Reichelt S, Owsianski-Hille N, Derlin T, Berding G, et al. A randomized, double-blind, crossover comparison of novel continuous bed motion versus traditional bed position whole-body PET/CT imaging. Eur J Nucl Med Mol Imaging. 2016;43(4):711-7. doi:10.1007/s00259-015-3226-z.

62. Acuff SN, Osborne D. Clinical workflow considerations for implementation of continuous-bed-motion PET/CT. J Nucl Med Technol. 2016;44(2):55-8. doi:10.2967/jnmt.116.172171.

63. Frach T, Prescher G, Degenhardt C, de Gruyter R, Schmitz A, Ballizany R, editors. The digital silicon photomultiplier-principle of operation and intrinsic detector performance. New York: Nuclear Science Symposium Conference Record (NSS/MIC): IEEE; 2009.

64. Degenhardt C, Prescher G, Frach T, Thon A, de Gruyter R, Schmitz A, et al., editors. The digital silicon photomultiplier - a novel sensor for the detection of scintillation light. New York: Nuclear Science Symposium Conference Record (NSS/MIC): IEEE; 2009.

65. Degenhardt C, Rodrigues P, Trindade A, Zwaans B, Mülhens O, Dorscheid R, et al., editors. Performance evaluation of a prototype positron emission tomography scanner using digital photon counters (DPC). New York: Nuclear Science Symposium and Medical Imaging Conference (NSS/MIC): IEEE; 2012.

66. Narayanan M, Andreyev A, Bai C, Miller M, Hu Z. TOF-benefits on the Philips digital PET/CT scanner: evaluation of faster convergence and reduced scan times. J Nucl Med. 2016;57(supplement 2):201.

67. Zhang J, Binzel K, Bardos P, Nagar V, Knopp M, Zhang B, et al. FDG dose reduction potential of a next generation digital detector PET/CT system: initial clinical demonstration in whole-body imaging. J Nucl Med. 2015;56(supplement 3):1823.

68. Nguyen NC, Vercher-Conejero JL, Sattar A, Miller MA, Maniawski PJ, Jordan DW, et al. Image quality and diagnostic performance of a digital PET prototype in patients with oncologic diseases: initial experience and comparison with analog PET. J Nucl Med. 2015;56(9):1378-85. doi:10.2967/jnumed.114.148338.

69. Hofmann M, Pichler B, Scholkopf B, Beyer T. Towards quantitative PET/MRI: a review of MR-based attenuation correction techniques. Eur J Nucl Med Mol Imaging. 2009;36(supplement 1):S93104. doi:10.1007/s00259-008-1007-7.

70. Mehranian A, Zaidi H. Emission-based estimation of lung attenuation coefficients for attenuation correction in time-of-flight PET/ MR. Phys Med Biol. 2015;60(12):4813-33. doi:10.1088/00319155/60/12/4813.

71. Benoit D, Ladefoged CN, Rezaei A, Keller SH, Andersen FL, Hojgaard L, et al. Optimized MLAA for quantitative non-TOF PET/MR of the brain. Phys Med Biol. 2016;61(24):8854-74. doi: 10.1088/1361-6560/61/24/8854.

72. Cheng JC, Salomon A, Yaqub M, Boellaard R. Investigation of practical initial attenuation image estimates in TOF-MLAA reconstruction for PET/MR. Med Phys. 2016;43(7):4163-73. doi:10. 1118/1.4953634.

73. Boellaard R, Quick HH. Current image acquisition options in PET/ MR. Semin Nucl Med. 2015;45(3):192-200. doi:10.1053/j. semnuclmed.2014.12.001.

74. Lasnon C, Salomon T, Desmonts C, Do P, Oulkhouir Y, Madelaine J, et al. Generating harmonized SUV within the EANM EARL accreditation program: software approach versus EARL-compliant reconstruction. Ann Nucl Med. 2017;31(2):125-34. doi:10.1007/ s12149-016-1135-2. 
75. Quak E, Le Roux PY, Hofman MS, Robin P, Bourhis D, Callahan J, et al. Harmonizing FDG PET quantification while maintaining optimal lesion detection: prospective multicentre validation in 517 oncology patients. Eur J Nucl Med Mol Imaging. 2015;42(13): 2072-82. doi:10.1007/s00259-015-3128-0.

76. Lasnon C, Desmonts C, Quak E, Gervais R, Do P, Dubos-Arvis C, et al. Harmonizing SUVs in multicentre trials when using different generation PET systems: prospective validation in non-small cell lung cancer patients. Eur J Nucl Med Mol Imaging. 2013;40(7): 985-96. doi:10.1007/s00259-013-2391-1.

77. Aide N, Lasnon C, Veit Haibach P, Sera T, Sattler B, Boellaard R. EANM/EARL harmonization strategies in PET quantification: from daily practice to multicentre oncological studies. Eur J Nucl Med Mol Imaging. 2017. doi:10.1007/s00259-017-3740-2.

78. de Groot EH, Post N, Boellaard R, Wagenaar NR, Willemsen AT, van Dalen JA. Optimized dose regimen for whole-body FDG-PET imaging. EJNMMI Res. 2013;3(1):63. doi:10.1186/2191-219X-3-63.

79. Koopman D, van Osch JA, Jager PL, Tenbergen CJ, Knollema S, Slump CH, et al. Technical note: how to determine the FDG activity for tumour PET imaging that satisfies European guidelines. EJNMMI Phys. 2016;3(1):22. doi:10.1186/s40658-016-0158-z.

80. Boellaard R, O'Doherty MJ, Weber WA, Mottaghy FM, Lonsdale $\mathrm{MN}$, Stroobants SG, et al. FDG PET and PET/CT: EANM procedure guidelines for tumour PET imaging: version 1.0. Eur J Nucl Med Mol Imaging. 2010;37(1):181-200. doi:10.1007/s00259-009-1297-4.

81. Selwyn RG, Nickles RJ, Thomadsen BR, DeWerd LA, Micka JA. A new internal pair production branching ratio of $90 \mathrm{Y}$ : the development of a non-destructive assay for $90 \mathrm{Y}$ and $90 \mathrm{Sr}$. Appl Radiat Isot. 2007;65(3):318-27. doi:10.1016/j.apradiso.2006.08.009.

82. Zade AA, Rangarajan V, Purandare NC, Shah SA, Agrawal AR, Kulkarni SS, et al. 90Y microsphere therapy: does 90Y PET/CT imaging obviate the need for $90 \mathrm{Y}$ bremsstrahlung SPECT/CT imaging? Nucl Med Commun. 2013;34(11):1090-6. doi:10.1097/ MNM.0b013e328364aa4b.

83. Pasciak AS, Bourgeois AC, Bradley YC. A comparison of techniques for $90 \mathrm{Y}$ PET/CT image-based dosimetry following radioembolization with resin microspheres. Front Oncol. 2014;4: 121. doi:10.3389/fonc.2014.00121.

84. Willowson KP, Tapner M, Team QI, Bailey DL. A multicentre comparison of quantitative $90 \mathrm{Y} \mathrm{PET/CT} \mathrm{for} \mathrm{dosimetric} \mathrm{purposes}$ after radioembolization with resin microspheres: the QUEST phantom study. Eur J Nucl Med Mol Imaging. 2015;42(8):1202-22. doi: 10.1007/s00259-015-3059-9.

85. Braat AJ, Smits ML, Braat MN, van den Hoven AF, Prince JF, de Jong HW, et al. 90 Y hepatic radioembolization: an update on current practice and recent developments. J Nucl Med. 2015;56(7): 1079-87. doi:10.2967/jnumed.115.157446.

86. Wright C, Binzel K, Zhang J, Wuthrick E, Tung C-H, Knopp M. Post-radioembolization assessment of intrahepatic yttrium-90 microsphere biodistribution using next-generation digital PET/CT and comparison to current pre/post-radioembolization SPECT/CT methodologies. J Nucl Med. 2016;57(supplement 2):197.

87. Preylowski V, Schlogl S, Schoenahl F, Jorg G, Samnick S, Buck $\mathrm{AK}$, et al. Is the image quality of I-124-PET impaired by an automatic correction of prompt gammas? PLoS One. 2013;8(8):e71729. doi:10.1371/journal.pone.0071729.

88. Lubberink M, Herzog H. Quantitative imaging of 124I and 86Y with PET. Eur J Nucl Med Mol Imaging. 2011;38(supplement 1): S10-8. doi:10.1007/s00259-011-1768-2.

89. Surti S, Scheuermann R, Karp JS. Correction technique for cascade gammas in I-124 imaging on a fully-3D, time-of-flight PET scanner. IEEE Trans Nucl Sci. 2009;56(3):653-60. doi:10.1109/TNS. 2008.2011805.

90. Makris NE, Boellaard R, Visser EP, de Jong JR, Vanderlinden B, Wierts R, et al. Multicenter harmonization of $89 \mathrm{Zr}$ PET/CT performance. J Nucl Med. 2014;55(2):264-7. doi:10.2967/jnumed.113. 130112.

91. Conti M, Eriksson L. Physics of pure and non-pure positron emitters for PET: a review and a discussion. EJNMMI Phys. 2016;3(1): 8. doi:10.1186/s40658-016-0144-5. 